\title{
A POSTERIORI ERROR ESTIMATES FOR THE FINITE ELEMENT APPROXIMATION OF EIGENVALUE PROBLEMS
}

\author{
RICARDO G. DURÁN* \\ Departamento de Matemática, Facultad de Ciencias Exactas y Naturales \\ Universidad de Buenos Aires, 1428 Buenos Aires, Argentina \\ E-mail: rduran@dm.uba.ar \\ CLAUDIO PADRA* \\ Centro Atómico Bariloche, 8400 Bariloche, Río Negro, Argentina \\ E-mail: padra@cab.cnea.gov.ar \\ RODOLFO RODRÍGUEZ \\ $G I^{2} M A$, Departamento de Ingeniería Matemática \\ Universidad de Concepción, Casilla 160-C, Concepción, Chile \\ E-mail: rodolfo@ing-mat.udec.cl
}

\begin{abstract}
This paper deals with a posteriori error estimators for the linear finite element approximation of second order elliptic eigenvalue problems in two or three dimensions. First, we give a simple proof of the equivalence, up to higher order terms, between the error and a residual type error estimator. Second, we prove that the volumetric part of the residual is dominated by a constant times the edge or face residuals, again up to higher order terms. This result was not known for eigenvalue problems.
\end{abstract}

Keywords: Eigenvalue problems, Finite elements, A posteriori error estimates.

\section{Introduction}

In many applications it is important to find the eigenvalues and eigenfunctions of an elliptic partial differential equation. Finite element methods for these problems have been widely used and analyzed under a general framework in many works. We refer to Babuška and Osborn, ${ }^{4}$ Raviart and Thomas, ${ }^{9}$ Strang and Fix, ${ }^{13}$ and their references, where convergence and optimal error estimates for both eigenvalues and eigenfunctions are obtained.

In numerical computations it is important to use adaptive procedures based on a posteriori error estimators. Several approaches have been considered to construct estimators based on the residual equations (see the books by Ainsworth and Oden ${ }^{1}$ and Verfürth, ${ }^{14}$ and their references). For eigenvalue problems, Verfürht ${ }^{14,15}$ obtained results based on a general analysis for non-linear equations. Also, Larson ${ }^{7}$ analyzed this problem obtaining similar results by using a different approach.

${ }^{*}$ Member of CONICET, Argentina. 
In the present paper, we give a simpler analysis for a residual type error estimator for the linear finite element approximations of a model eigenvalue problem. We prove that the estimator is equivalent to the error up to higher order terms. We remark that in the above mentioned papers by Larson ${ }^{7}$ and Verfürth ${ }^{15}$, the $a$ posteriori error estimates are obtained assuming that the numerical solution is close enough to the exact one. We do not assume this and that is why we have to add explicit higher order terms in our reliability and efficiency estimates. If we neglect the higher order terms, the constants appearing in the equivalence are independent of the eigenvalue (they depend only on the regularity of the meshes). The constants in front of the higher order terms depend also on the eigenvalue being approximated.

The second goal of this paper is to analyze a simpler error estimator based only on the jumps of the normal derivative of the approximate solution, which turns out to be equivalent to the error also up to higher order terms. Let us remark that, in particular, this result allows proving the equivalence between the error and a Zienkiewicz-Zhu type error estimator (see Rodríguez ${ }^{12}$ ), which is the most used by engineers. In this way, results that are well known for source problems can be generalized to eigenvalue problems (see, for example, Babuška and Miller, ${ }^{3}$ Carstensen and Verfürth ${ }^{5}$, Nochetto, ${ }^{8}$ and Rodríguez ${ }^{12}$ ).

The rest of the paper is organized as follows. In Section 2 we introduce the model problem and recall some known a priori error estimates for its finite element approximation. In Section 3 we define an error estimator and prove its equivalence with the error up to higher order terms. Finally, in Section 4, we prove that the edge or face residuals dominate the other part of the estimator and, therefore, a simpler equivalent error estimator is obtained.

\section{Model problem and finite element approximation}

Let $\Omega \subset \mathbb{R}^{d}$ be a polygonal domain for $d=2$ and a polyhedral domain for $d=3$. We consider the following model problem:

Seek a real number $\lambda$ and a non-zero real-valued function u satisfying

$$
\left\{\begin{array}{cl}
-\Delta u=\lambda u & \text { in } \Omega \\
u=0 & \text { on } \partial \Omega .
\end{array}\right.
$$

We use standard notation for Sobolev spaces, norms, and seminorms. Hence, the eigenvalues $\lambda$ and normalized eigenfunctions $u$ of the problem above satisfy, $u \in H_{0}^{1}(\Omega)$ and,

$$
\left\{\begin{array}{l}
\int_{\Omega} \nabla u \cdot \nabla v=\lambda \int_{\Omega} u v \quad \forall v \in H_{0}^{1}(\Omega), \\
\int_{\Omega}|u|^{2}=1
\end{array}\right.
$$

It is well known that this problem attains a sequence of eigenpairs $\left(\lambda_{j}, u_{j}\right)$, with positive eigenvalues $\lambda_{j}$ diverging to $+\infty$. We assume the eigenvalues to be 
increasingly ordered: $\lambda_{1} \leq \cdots \leq \lambda_{j} \leq \cdots$. The associated eigenfunctions satisfy $u_{j} \in H^{1+r}(\Omega)$, for some $r \in(0,1]$ depending on $\Omega$ ( $r=1$ when $\Omega$ is convex).

We consider a family $\left\{\mathcal{T}_{h}\right\}$ of triangulations of $\Omega$, formed by triangles if $d=2$, or tetrahedra if $d=3$, such that any two elements in $\mathcal{T}_{h}$ share at most a vertex, an edge, or a face. Let $h$ stand for the mesh-size; namely $h=\max _{T \in \mathcal{T}_{h}} h_{T}$, with $h_{T}$ being the diameter of the element $T$. For each $\mathcal{T}_{h}$ we denote with $V_{h} \subset H_{0}^{1}(\Omega)$ the standard finite element space of continuous piecewise linear elements.

The finite element approximate solutions of our spectral problem are defined by $u_{h} \in V_{h}$ and,

$$
\left\{\begin{array}{l}
\int_{\Omega} \nabla u_{h} \cdot \nabla v_{h}=\lambda_{h} \int_{\Omega} u_{h} v_{h} \quad \forall v_{h} \in V_{h}, \\
\int_{\Omega}\left|u_{h}\right|^{2}=1 .
\end{array}\right.
$$

The problem above reduces to a generalized eigenvalue problem involving positive definite symmetric matrices. It attains a finite number of eigenpairs $\left(\lambda_{j h}, u_{j h}\right)$, $1 \leq j \leq N_{h}=\operatorname{dim} V_{h}$, with positive eigenvalues, which we also assume increasingly ordered: $\lambda_{1 h} \leq \cdots \leq \lambda_{N_{h} h}$.

The following a priori error estimates are well known (see for example Theorem 6.4-3 and Lemma 6.4-4 in Raviart and Thomas $\left.{ }^{9}\right)$. If $\left(\lambda_{j}, u_{j}\right)$ is a solution of (2.1), then there exists $\left(\lambda_{j h}, u_{j h}\right)$ satisfying $(2.2)$ such that

$$
\begin{aligned}
\left|u_{j}-u_{j h}\right|_{1, \Omega} & \leq C h^{r} \\
\| u_{j}-\left.u_{j h}\right|_{0, \Omega} & \leq C h^{r}\left|u_{j}-u_{j h}\right|_{1, \Omega}, \\
\left|\lambda_{j}-\lambda_{j h}\right| & \leq C\left|u_{j}-u_{j h}\right|_{1, \Omega}^{2},
\end{aligned}
$$

where, here and thereafter, $C$ denotes a positive constant, depending only on the particular eigenvalue and the regularity of $\mathcal{T}_{h}$.

\section{Error estimator}

In this section we introduce the error estimator and prove its equivalence with the error.

First we introduce some notation. Let $\mathcal{F}$ be the set of all interior faces of the mesh (i.e., faces of elements not lying on $\partial \Omega$ ) and $\mathcal{F}_{T} \subset \mathcal{F}$ be the subset of faces of $T$. For each face $F \in \mathcal{F}$ we choose an arbitrary unit normal vector $n_{F}$ and denote the two elements sharing this face $T_{\text {in }}$ and $T_{\text {out }}$, where $n_{F}$ points outwards $T_{\text {in }}$. For $v_{h} \in V_{h}$ we introduce the jump $J_{F}$ of the normal derivative of $v_{h}$ across the face $F$, namely,

$$
J_{F}=\nabla\left(\left.v_{h}\right|_{T_{\text {out }}}\right) \cdot n_{F}-\nabla\left(\left.v_{h}\right|_{T_{\text {in }}}\right) \cdot n_{F}
$$

Note that this value is independent of the choice of $n_{F}$.

We consider a particular eigenpair $\left(\lambda_{j}, u_{j}\right)$ and its corresponding finite element approximation $\left(\lambda_{j h}, u_{j h}\right)$. From now on, we drop out the subindices $j$ to simplify the notation. Our goal is to estimate the error $e=u-u_{h}$. 
The following lemmas provide some error equations which will be the starting points of our error analysis.

Lemma 3.1 For $v \in H_{0}^{1}(\Omega)$ there holds

$$
\int_{\Omega} \nabla e \cdot \nabla v-\int_{\Omega}\left(\lambda u-\lambda_{h} u_{h}\right) v=\sum_{T \in \mathcal{T}_{h}}\left(\int_{T} \lambda_{h} u_{h} v+\frac{1}{2} \sum_{F \in \mathcal{F}_{T}} \int_{F} J_{F} v\right) .
$$

Proof. Take $v \in H_{0}^{1}(\Omega)$. Integrating by parts in each element we have

$$
\begin{aligned}
\int_{\Omega} \nabla e \cdot \nabla v-\int_{\Omega}\left(\lambda u-\lambda_{h} u_{h}\right) v & =\sum_{T \in \mathcal{T}_{h}}\left(\int_{T} \lambda_{h} u_{h} v+\int_{\partial T} \frac{\partial e}{\partial n} v\right) \\
& =\sum_{T \in \mathcal{T}_{h}}\left(\int_{T} \lambda_{h} u_{h} v+\frac{1}{2} \sum_{F \in \mathcal{F}_{T}} \int_{F} J_{F} v\right) .
\end{aligned}
$$

Lemma 3.2 There holds

$$
\int_{\Omega}\left(\lambda u-\lambda_{h} u_{h}\right) e=\left(\lambda+\lambda_{h}\right)\left(1-\int_{\Omega} u u_{h}\right)=\frac{\lambda+\lambda_{h}}{2} \int_{\Omega} e^{2} .
$$

Proof. It follows easily by using that $\int_{\Omega}|u|^{2}=\int_{\Omega}\left|u_{h}\right|^{2}=1$.

Let us now define the local error indicator $\eta_{T}$ by

$$
\eta_{T}=\left(h_{T}^{2} \lambda_{h}^{2}\left\|u_{h}\right\|_{0, T}^{2}+\frac{1}{2} \sum_{F \in \mathcal{F}_{T}} h_{F}\left\|J_{F}\right\|_{0, F}^{2}\right)^{1 / 2},
$$

where $h_{F}$ denotes the diameter of the face $F$, and the global error estimator by

$$
\eta=\left(\sum_{T \in \mathcal{T}_{h}} \eta_{T}^{2}\right)^{1 / 2}
$$

As usual in residual-type error indicators, $\eta_{T}$ consists of two conveniently weighted terms: the $L^{2}$ norm of the volumetric residual, which in our problem reduces to $\lambda_{h}\left\|u_{h}\right\|_{0, T}$, and the $L^{2}$ norm of the jumps $J_{F}$ across the interior faces of the element.

Meshes generated for adaptive refinement in problems involving singularities are usually highly non-uniform (i.e., the elements of $\mathcal{T}_{h}$ may have very different sizes). However, if the refinement is done in a proper way, the family $\left\{\mathcal{T}_{h}\right\}$ can be constructed in such a way that the regularity is preserved (see for instance Arnold et al. ${ }^{2}$ Rivara, ${ }^{10}$ and Rivara and Vénere ${ }^{11}$ ). Therefore, it is natural to seek error estimates with constants depending only on the regularity of the meshes (and not on the element size). We will show that the estimator $\eta$ is of this kind.

The following theorem gives an upper error estimate.

Theorem 3.1 There exists a constant $C$, depending only on the regularity of $\mathcal{T}_{h}$, such that

$$
|e|_{1, \Omega} \leq C \eta+\left(\frac{\lambda+\lambda_{h}}{2}\right)^{\frac{1}{2}}\|e\|_{0, \Omega} .
$$


Proof. Let $e^{\mathrm{I}} \in V_{h}$ be such that

$$
\left\|e-e^{\mathrm{I}}\right\|_{0, T} \leq C h_{T}|e|_{1, \widetilde{T}}
$$

and

$$
\left\|e-e^{\mathrm{I}}\right\|_{0, F} \leq C h_{F}^{1 / 2}|e|_{1, \widetilde{T}},
$$

where $\widetilde{T}$ is the union of all the elements sharing a vertex with $T$. We can take, for example, the well known Clément average interpolant of $e$ (see, for example, Clément $\left.{ }^{6}\right)$.

Using Eqs. (2.1) and (2.2) we have

$$
\int_{\Omega}|\nabla e|^{2}=\int_{\Omega} \nabla e \cdot \nabla\left(e-e^{\mathrm{I}}\right)+\int_{\Omega}\left(\lambda u-\lambda_{h} u_{h}\right) e^{\mathrm{I}} .
$$

Hence, from Lemma 3.1 with $v=e-e^{\mathrm{I}}$, we have

$$
\int_{\Omega}|\nabla e|^{2}=\sum_{T \in \mathcal{T}_{h}}\left[\int_{T} \lambda_{h} u_{h}\left(e-e^{\mathrm{I}}\right)+\frac{1}{2} \sum_{F \in \mathcal{F}_{T}} \int_{F} J_{F}\left(e-e^{\mathrm{I}}\right)\right]+\int_{\Omega}\left(\lambda u-\lambda_{h} u_{h}\right) e,
$$

and using Lemma 3.2, Schwartz inequality, (3.1), and (3.2), we obtain

$$
\int_{\Omega}|\nabla e|^{2} \leq C \eta|e|_{1, \Omega}+\frac{\left(\lambda+\lambda_{h}\right)}{2}\|e\|_{0, \Omega}^{2},
$$

which allows us to conclude the proof.

Remark 3.1 As a consequence of the previous theorem and the a priori estimate (2.4), the global estimator provides an upper bound of the error in energy norm up to a multiplicative constant and a higher order term:

$$
|e|_{1, \Omega} \leq C\left(\eta+h^{r}|e|_{1, \Omega}\right) .
$$

Our next goal is to show that the local error indicators $\eta_{T}$ are efficient in the sense of pointing out which elements should be effectively refined because they support large local errors.

For $T \in \mathcal{T}_{h}$, let $b_{T} \in H_{0}^{1}(T)$ be the standard cubic bubble function, if $d=2$, or the standard quartic bubble function, if $d=3$, in both cases attaining the value one at the barycenter of $T$. Using scaling arguments as those used to prove standard local inverse inequalities one can see that there exists a constant $C$, which only depends on the regularity of the element $T$, such that

$$
\left\|u_{h} b_{T}\right\|_{0, T} \leq\left\|u_{h}\right\|_{0, T} \leq C\left(\int_{T}\left|u_{h}\right|^{2} b_{T}\right)^{1 / 2}
$$

and

$$
\left|u_{h} b_{T}\right|_{1, T} \leq \frac{C}{h_{T}}\left\|u_{h}\right\|_{0, T} .
$$

The following lemma provides an upper estimate of the volumetric residual term. 
R. G. Durán, C. Padra, and R. Rodríguez

Lemma 3.3 There exists a constant $C$, depending only on the regularity of $T$, such that

$$
h_{T} \lambda_{h}\left\|u_{h}\right\|_{0, T} \leq C\left(|e|_{1, T}+h_{T}\left\|\lambda u-\lambda_{h} u_{h}\right\|_{0, T}\right) .
$$

Proof. Take $v=u_{h} b_{T}$. Using (2.1) and the fact that $\int_{T} \nabla u_{h} \cdot \nabla v=0$, we obtain

$$
\lambda_{h} \int_{T} u_{h} v=\int_{T} \nabla e \cdot \nabla v-\int_{T}\left(\lambda u-\lambda_{h} u_{h}\right) v
$$

and the lemma follows easily by using (3.3) and (3.4).

Now, for $F \in \mathcal{F}$, let $T_{F}^{1}$ and $T_{F}^{2}$ be the two elements in $\mathcal{T}_{h}$ sharing $F$ and let $b_{F} \in H_{0}^{1}\left(T_{F}^{1} \cup T_{F}^{2}\right)$ be the piecewise quadratic function, if $d=2$, or cubic function, if $d=3$, in both cases attaining the value 1 at the barycenter of $F$. Then, using again scaling arguments, it is easy to see that there exists a constant $C$ such that

$$
\left\|b_{F}\right\|_{0, T_{F}^{i}} \leq C h_{F}^{d / 2} \quad \text { and } \quad\left|b_{F}\right|_{1, T_{F}^{i}} \leq \frac{C}{h_{T_{F}^{i}}}\left\|b_{F}\right\|_{0, T_{F}^{i}}, \quad i=1,2 .
$$

The following lemma provides an upper estimate for the jump terms of the local error indicator.

Lemma 3.4 There exists a constant $C$, depending only on the regularity of $T_{F}^{1}$ and $T_{F}^{2}$, such that

$$
h_{F}^{1 / 2}\left\|J_{F}\right\|_{0, F} \leq C\left(|e|_{1, T_{F}^{1} \cup T_{F}^{2}}+h_{F}\left\|\lambda u-\lambda_{h} u_{h}\right\|_{0, T_{F}^{1} \cup T_{F}^{2}}\right) .
$$

Proof. For any $v \in H_{0}^{1}\left(T_{F}^{1} \cup T_{F}^{2}\right)$ it follows from Lemma 3.1 that

$$
\int_{F} J_{F} v=\int_{T_{F}^{1} \cup T_{F}^{2}} \nabla e \cdot \nabla v-\int_{T_{F}^{1} \cup T_{F}^{2}}\left(\lambda u-\lambda_{h} u_{h}\right) v-\lambda_{h} \int_{T_{T}^{1} \cup T_{F}^{2}} u_{h} v .
$$

Taking $v=b_{F}$ in this equation and using the fact that $J_{F}$ is constant together with Lemma 3.3, we conclude the proof.

Now we are in order to prove an upper estimate of the local error indicator.

Theorem 3.2 Let $T^{*}$ be the union of $T$ and the neighboring elements $T^{\prime}$ sharing a face with $T$. There exists a positive constant $C$, depending only on the regularity of the elements of $T^{*}$, such that

$$
\eta_{T} \leq C\left(|e|_{1, T^{*}}+h_{T}\left\|\lambda u-\lambda_{h} u_{h}\right\|_{0, T^{*}}\right) .
$$

Proof. It follows immediately from Lemmas 3.3 and 3.4.

Remark 3.2 The term $h_{T}\left\|\lambda u-\lambda_{h} u_{h}\right\|_{0, T^{*}}$ in the previous theorem is a higher order term. In fact, for each $T^{\prime} \in \mathcal{T}_{h}$,

$$
\begin{aligned}
h_{T^{\prime}}\left\|\lambda u-\lambda_{h} u_{h}\right\|_{0, T^{\prime}} & \leq \frac{\left|\lambda-\lambda_{h}\right|}{\left|\lambda_{h}\right|} \lambda_{h} h_{T^{\prime}}\left\|u_{h}\right\|_{0, T^{\prime}}+\lambda h_{T^{\prime}}\left\|u-u_{h}\right\|_{0, T^{\prime}} \\
& \leq C h^{2 r} \eta_{T^{\prime}}+\lambda h_{T^{\prime}}\|e\|_{0, T^{\prime}}
\end{aligned}
$$


the last inequality because of (2.5) and the definition of $\eta_{T^{\prime}}$. Note that the first term in the right hand side is asymptotically negligible with respect to the local indicator $\eta_{T^{\prime}}$, whereas the second term is asymptotically negligible with respect to the local error $\|e\|_{1, T^{\prime}}$.

Consequently, the theorem above shows that the error indicator of an element $T$ is bounded above by the energy error on $T^{*}$, up to a multiplicative constant, and higher order terms, namely,

$$
\eta_{T} \leq C|e|_{1, T^{*}}+\mathcal{O}\left(h^{2 r}\right) \eta_{T^{*}}+\mathcal{O}\left(h_{T}\right)\|e\|_{0, T^{*}} .
$$

where, $\eta_{T^{*}}^{2}=\sum_{T \in T^{*}} \eta_{T}^{2}$.

Therefore, $\eta_{T}$ is an efficient error indicator, in the sense that when $\eta_{T}$ is large, the error in the vicinity of the element $T$ must be large too. Thus, $\eta_{T}$ points out correctly the elements which should be refined, at least when the global term $\frac{\left|\lambda-\lambda_{h}\right|}{\left|\lambda_{h}\right|}$ is small enough.

\section{A face residual error estimator}

The goal of this section is to define a new error indicator not involving the volumetric part of $\eta_{T}$. To do this, we will prove that the face residuals dominate the volumetric ones up to higher order terms.

Let $\mathcal{N}$ be the set of interior vertices of the triangulation $\mathcal{T}_{h}$ (i.e., vertices $P \notin$ $\partial \Omega)$. For $P \in \mathcal{N}$, let $\Omega_{P}=\bigcup\left\{T \in \mathcal{T}_{h}: P \in T\right\}$. Let $\varphi_{P} \in V_{h}$ be the basis function with $\operatorname{supp} \varphi_{P}=\Omega_{P}$ and $h_{P}$ the diameter of $\Omega_{P}$. Finally, let $\mathcal{F}_{P} \subset \mathcal{F}$ be the subset of faces containing the vertex $P$ and $\mathcal{T}_{P}=\left\{T \in \mathcal{T}_{h}: T \ni P\right\}$.

The following relation between the basis functions and the jumps is used in the sequel.

Lemma 4.1 For all $P \in \mathcal{N}$, there holds

$$
\lambda_{h} \int_{\Omega_{P}} u_{h} \varphi_{P}=\sum_{F \in \mathcal{F}_{P}} \frac{\operatorname{meas}(F)}{d} J_{F} .
$$

Proof. Using (2.2) and integrating by parts we have

$$
\lambda_{h} \int_{\Omega_{P}} u_{h} \varphi_{P}=\int_{\Omega} \nabla u_{h} \cdot \nabla \varphi_{P}=\sum_{F \in \mathcal{F}_{P}} \frac{\operatorname{meas}(F)}{d} J_{F} .
$$

Our next step is to prove a local estimate for the volumetric part of the residual.

Lemma 4.2 For all $P \in \mathcal{N}$, there holds

$$
\sum_{T \in \mathcal{T}_{P}} h_{T}^{2} \lambda_{h}^{2}\left\|u_{h}\right\|_{0, T}^{2} \leq C\left(\sum_{F \in \mathcal{F}_{P}} h_{F}\left\|J_{F}\right\|_{0, F}^{2}+h_{P}^{4} \lambda_{h}^{2}\left\|\nabla u_{h}\right\|_{0, \Omega_{P}}^{2}\right),
$$

where $C$ is a constant depending only on the regularity of the mesh. 
Proof. Let $u_{h}^{P}$ denote the $L^{2}\left(\Omega_{P}\right)$ projection of $u_{h}$ onto the constants. Then, we have

$$
\sum_{T \in \mathcal{T}_{P}} h_{T}^{2} \lambda_{h}^{2}\left\|u_{h}\right\|_{0, T}^{2} \leq h_{P}^{2} \lambda_{h}^{2}\left\|u_{h}\right\|_{0, \Omega_{P}}^{2}=h_{P}^{2} \lambda_{h}^{2}\left(\left\|u_{h}-u_{h}^{P}\right\|_{0, \Omega_{P}}^{2}+\left\|u_{h}^{P}\right\|_{0, \Omega_{P}}^{2}\right) .
$$

Now,

$$
\begin{aligned}
\left\|u_{h}^{P}\right\|_{0, \Omega_{P}}^{2} & =\frac{(d+1)^{2}}{\operatorname{meas}\left(\Omega_{P}\right)}\left(\int_{\Omega_{P}} u_{h}^{P} \varphi_{P}\right)^{2} \\
& \leq \frac{2(d+1)^{2}}{\operatorname{meas}\left(\Omega_{P}\right)}\left\{\left[\int_{\Omega_{P}}\left(u_{h}^{P}-u_{h}\right) \varphi_{P}\right]^{2}+\left(\int_{\Omega_{P}} u_{h} \varphi_{P}\right)^{2}\right\} .
\end{aligned}
$$

Then, using Lemma 4.1 and Cauchy-Schwartz in the previous estimate, we have

$$
\left\|u_{h}^{P}\right\|_{0, \Omega_{P}}^{2} \leq C\left(\left\|u_{h}-u_{h}^{P}\right\|_{0, \Omega_{P}}^{2}+\frac{1}{\lambda_{h}^{2} \operatorname{meas}\left(\Omega_{P}\right)} \sum_{F \in \mathcal{F}_{P}} h_{F}^{d-1}\left\|J_{F}\right\|_{0, F}^{2}\right) .
$$

Thus, substituting the expression above in (4.1), using the standard estimate for the $L^{2}\left(\Omega_{P}\right)$ projection and the fact that $h_{F}, h_{P}$ and meas $\left(\Omega_{P}\right)^{1 / d}$ are all equivalent, we conclude the proof.

Now we are in order to define the simpler indicator

$$
\tilde{\eta}_{T}=\left(\frac{1}{2} \sum_{F \in \mathcal{F}_{T}} h_{F}\left\|J_{F}\right\|_{0, F}^{2}\right)^{1 / 2},
$$

and the corresponding global error estimator

$$
\tilde{\eta}=\left(\sum_{T \in \mathcal{T}_{h}} \tilde{\eta}_{T}^{2}\right)^{1 / 2}
$$

The following theorem shows that this estimator is globally reliable and locally efficient up to higher order terms.

Theorem 4.1 There exists a constant $C$, depending only on the regularity of $\mathcal{T}_{h}$, such that

$$
|e|_{1, \Omega} \leq C\left[\tilde{\eta}+\left(\frac{\lambda+\lambda_{h}}{2}\right)^{\frac{1}{2}}\|e\|_{0, \Omega}+\lambda_{h}^{3 / 2} h^{2}\right]
$$

and

$$
\tilde{\eta}_{T} \leq C\left(|e|_{1, T^{*}}+h_{T}\left\|\lambda u-\lambda_{h} u_{h}\right\|_{0, T^{*}}\right) .
$$

Proof. Theorem 3.2 yields the second estimate above, whereas Theorem 3.1 leads to

$$
|e|_{1, \Omega}^{2} \leq C\left(\tilde{\eta}^{2}+\sum_{T \in \mathcal{T}_{h}} h_{T}^{2} \lambda_{h}^{2}\left\|u_{h}\right\|_{0, T}^{2}+\frac{\lambda+\lambda_{h}}{2}\|e\|_{0, \Omega}^{2}\right) .
$$


From Lemma 4.2 we have

$$
\sum_{T \in \mathcal{T}_{h}} h_{T}^{2} \lambda_{h}^{2}\left\|u_{h}\right\|_{0, T}^{2} \leq C\left(\tilde{\eta}^{2}+h^{4} \lambda_{h}^{2}\left\|\nabla u_{h}\right\|_{0, \Omega}^{2}\right) .
$$

Then, we conclude the proof from these two estimates and (2.2).

Remark 4.1 The first estimate in the theorem above involves an additional term $\lambda_{h}^{3 / 2} h^{2}$ which, under very mild assumptions, is also of higher order. Indeed, since the eigenfunctions of the Laplace operator are analytic in $\Omega,-\Delta u=\lambda u$ cannot vanish identically in any open subset. Therefore, according to Lemma 1.5.4 from Babuška and Miller, ${ }^{3}$ if there exist a disc $D \subset \Omega$ and a constant $\kappa>0$ such that

$$
\min _{\substack{T \in \mathcal{T}_{h} \\ T \subset \bar{D}}} h_{T} \geq \kappa h \quad \forall \mathcal{T}_{h}
$$

then there exists a constant $c>0$ such that $|e|_{1, \Omega} \geq c h$. Hence, in this case, the term $\lambda_{h}^{3 / 2} h^{2}$ is asymptotically negligible with respect to $|e|_{1, \Omega}$. Notice that the local quasi-uniformity property (4.2) holds in all practical cases.

The result of the previous theorem can be improved for the lowest eigenvalue. Indeed, the corresponding eigenfunction satisfies $u>0$, and so it is reasonable to assume that also $u_{h}>0$. (This is usually the case in practice; for instance, it is always true in $2 \mathrm{D}$ when the angles of all the triangles are not greater than $\frac{\pi}{2}$ ). In this case, the term $\lambda_{h}^{3 / 2} h^{2}$ can be dropped out from the reliability estimate. This is an immediate consequence of the following lemma, which shows that the volumetric residuals are dominated by the jumps when $u_{h}>0$.

Lemma 4.3 If $u_{h}>0$, then there exists a constant $C>0$, depending only on the regularity of $\mathcal{T}_{h}$, such that

$$
\sum_{T \in \mathcal{T}_{h}} \lambda_{h}^{2} h_{T}^{2}\left\|u_{h}\right\|_{0, T}^{2} \leq C \sum_{F \in \mathcal{F}} h_{F}\left\|J_{F}\right\|_{0, F}^{2} .
$$

Proof. We have

$$
\begin{aligned}
\sum_{T \in \mathcal{T}_{h}} \lambda_{h}^{2} h_{T}^{2}\left\|u_{h}\right\|_{0, T}^{2} & =\sum_{T \in \mathcal{T}_{h}} \int_{T} \lambda_{h}^{2} h_{T}^{2} u_{h}\left[\sum_{P \in \mathcal{N}} u_{h}(P) \varphi_{P}\right] \\
& =\sum_{P \in \mathcal{N}} u_{h}(P)\left(\sum_{T \in \mathcal{T}_{h}} \lambda_{h}^{2} h_{T}^{2} \int_{T} u_{h} \varphi_{P}\right) \\
& \leq \sum_{P \in \mathcal{N}} \lambda_{h} h_{P}^{2} u_{h}(P)\left(\lambda_{h} \int_{\Omega} u_{h} \varphi_{P}\right) \\
& \leq \sum_{P \in \mathcal{N}} \lambda_{h} h_{P}^{1+d / 2} u_{h}(P)\left[h_{P}^{1-d / 2} \sum_{F \in \mathcal{F}_{P}} \frac{\operatorname{meas}(F)}{d}\left|J_{F}\right|\right] \\
& \leq C\left[\sum_{P \in \mathcal{N}} \lambda_{h}^{2} h_{P}^{2+d}\left|u_{h}(P)\right|^{2}\right]^{1 / 2}\left(\sum_{F \in \mathcal{F}} h_{F}\left\|J_{F}\right\|_{0, F}^{2}\right)^{1 / 2},
\end{aligned}
$$


where we have used that $u_{h}>0$ for the first inequality and Lemma 4.1 for the second one. For the last one, we have used Cauchy-Schwartz inequality, twice, and the regularity of the mesh.

On the other hand, standard scaling arguments yield the following inequality

$$
h_{T}^{d} \sum_{P \in \mathcal{N}_{T}}\left|u_{h}(P)\right|^{2} \leq C\left\|u_{h}\right\|_{0, T}^{2}
$$

Then, we have

$$
\sum_{P \in \mathcal{N}} h_{P}^{2+d}\left|u_{h}(P)\right|^{2} \leq C \sum_{T \in \mathcal{T}_{h}} h_{T}^{2+d} \sum_{P \in \mathcal{N}_{T}}\left|u_{h}(P)\right|^{2} \leq C \sum_{T \in \mathcal{T}_{h}} h_{T}^{2}\left\|u_{h}\right\|_{0, T}^{2},
$$

which allows us to conclude the proof.

\section{Acknowledgments}

Ricardo G. Durán was partially supported by ANPCyT under grant PICT 03-05009 and by CONICET under grant PIP 0660/98. Claudio Padra was partially supported by ANPCyT under grant PICT 12-03239. Claudio Padra and Rodolfo Rodríguez were partially supported by FONDAP in Applied Mathematics.

\section{References}

1. M. Ainsworth, J. T. Oden, A Posteriori Error Estimation in Finite Element Analysis, (Wiley, 2000).

2. D. N. Arnold, A. Mukherjee, L. Pouly, Locally adapted tetrahedral meshes using bisection, SIAM J. Sci. Comput. 22 (2000) 431-448.

3. I. Babuška, A. Miller, A feedback finite element method with a posteriori error estimation. Part I: The finite element method and some basic properties of the a posteriori error estimator, Comp. Methods Appl. Mech. Engng. 61 (1987) 1-40.

4. I. Babuška, J. Osborn, Eigenvalue Problems, in Handbook of Numerical Analysis, Vol. II, eds. P. G. Ciarlet and J. L. Lions (North Holland, 1991) pp. 641-787.

5. C. Carstensen, R. Verfürth, Edge residuals dominate a posteriori error estimates for low order finite element methods, SIAM J. Numer. Anal. 36 (1999) 1571-1587.

6. P. Clément, Approximation by finite element functions using local regularization, RAIRO R-2 (1975) 77-84.

7. M. G. Larson, A posteriori and a priori error analysis for finite element approximations of self-adjoint elliptic eigenvalue problems, SIAM J. Numer. Anal. 38 (2000) 608-625.

8. R. Nochetto, Pointwise a posteriori error estimates for elliptic problems on highly graded meshes, Math. Comp. 64 (1995) 1-22.

9. P. A. Raviart, J. M. Thomas, Introduction à l'Analyse Numérique des Equations aux Dérivées Partielles (Masson, 1983).

10. M. C. Rivara, Mesh refinement processes based on the generalized bisection of simplices, SIAM J. Numer. Anal. 21 (1984) 604-613.

11. M. C. Rivara, M. J. Vénere, Cost analysis of the longest-side refinement algorithm for triangulations, Eng. Comput. 12 (1996) 224-234.

12. R. Rodríguez, Some remarks on Zienkiewicz-Zhu estimator, Numer. Meth. PDEs. 10 (1994) 625-635. 
13. G. Strang, G. J. Fix, An Analysis of the Finite Element Method (Prentice Hall, 1973).

14. R. Verfürth, A Review of A Posteriori Error Estimation and Adaptive MeshRefinement Techniques (Wiley \& Teubner, 1996).

15. R. Verfürth, A posteriori error estimates for nonlinear problems, Math. Comp. 62 (1989) 445-475. 